\title{
Homogeneous Differential Equations Reducible to Separable Variables with GeoGebra
}

\author{
Jorge Olivares -Funes *and Pablo Martin and Maria Rojas Medar \\ Universidad de Antofagasta, Chile \\ * Corresponding Author
}

\begin{abstract}
.
Differential equations with GeoGebra are a topic that it is currently beginning to be taken with great enthusiasm in the development of support material for the teaching of this subject in engineering courses at various universities in the world, Its use in various mathematics courses especially in differential equations at the University of Antofagasta, dates back to 2018, where GeoGebra applets were created to see the animated solutions of first-order differential equations. This gave rise to the publication of "Animations and interactive creations in linear differential equations of first order: the case of Geogebra".This work is presented as a continuation and extension of this paper, here various examples are developed precisely with GeoGebra applets, this will be as the teaching support material in certain special cases of differential equations, which are reducible to separable variables, this is a case of complexity among the students, but thanks to this great software a very different and interactive approach is made known.
\end{abstract}

Keywords: Education; differential equations; GeoGebra, reduction; Tics

\section{Introduction}

Let us consider, first, the so called

$$
\frac{d y}{d x}=F\left(\frac{y}{x}\right)
$$

homogeneous differential equations, reducible to separables variables.

We will first show some examples tuve presented in the classes, which thanks to the support of GeoGebra, they will help in the motivation and disposition of the students towards learning the differential equations. 


\section{GeoGebra in homogeneous reducible differential equations}

Below we will show through GeoGebra examples of homogeneous reducible differential equations of the first order and their solutions, will be shown which can be downloaded or viewed online in https://www.geogebra.org/m/xqsvezyg .

\section{Example 1}

$\mathrm{Be}$

$$
\frac{d y}{d x}=\frac{a x-y}{x+y}
$$

whose solution in blue is show in Figure 1, and $a x^{2}+2 x y+y^{2}=c$ where "a" and "c" move between (-5) and 5

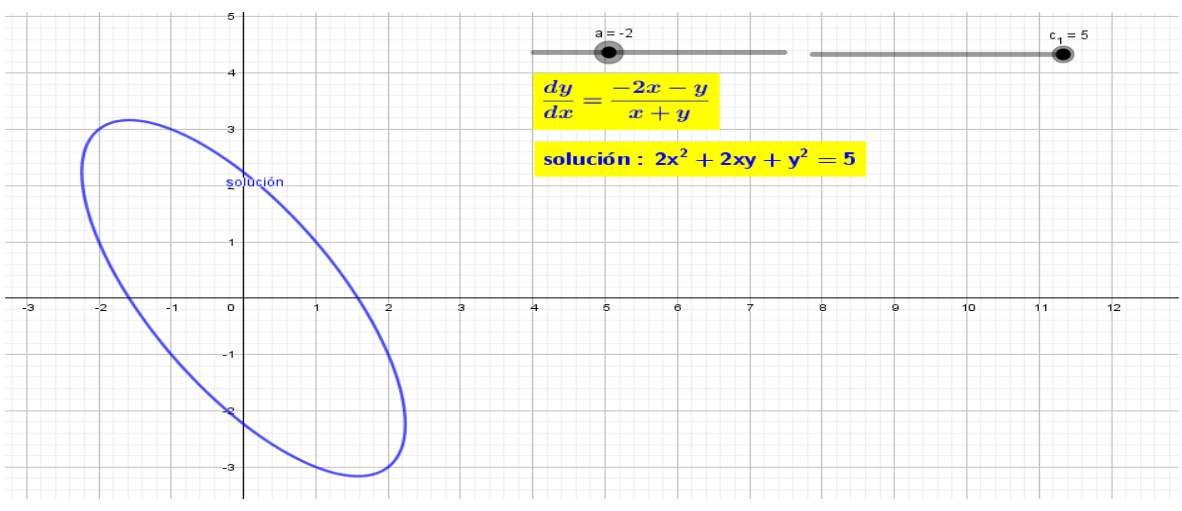

Figure 1

Example 2

$\mathrm{Be}$

$$
x \frac{d y}{d x}=\sqrt{x^{2}-y^{2}}+y
$$

whose solution in blue is shown in Figure 2 , and of is $y=x \operatorname{sen}(\ln (\mathrm{cx}))$ where "c" move between 0.1 and 5 .

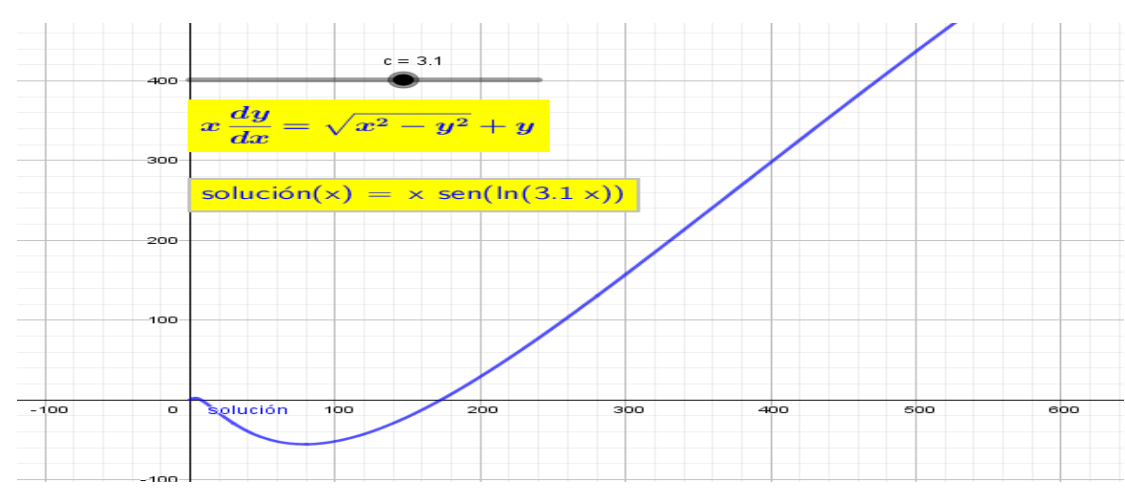

Figure 2 
Example 3

$\mathrm{Be}$

$$
\frac{d y}{d x}=\frac{y}{x}-1
$$

whose solution is shown in blue in Figure 3 , and $\mathrm{y}=\mathrm{c} \cdot x-x \ln x$ where "c" move between (-5) and 5.

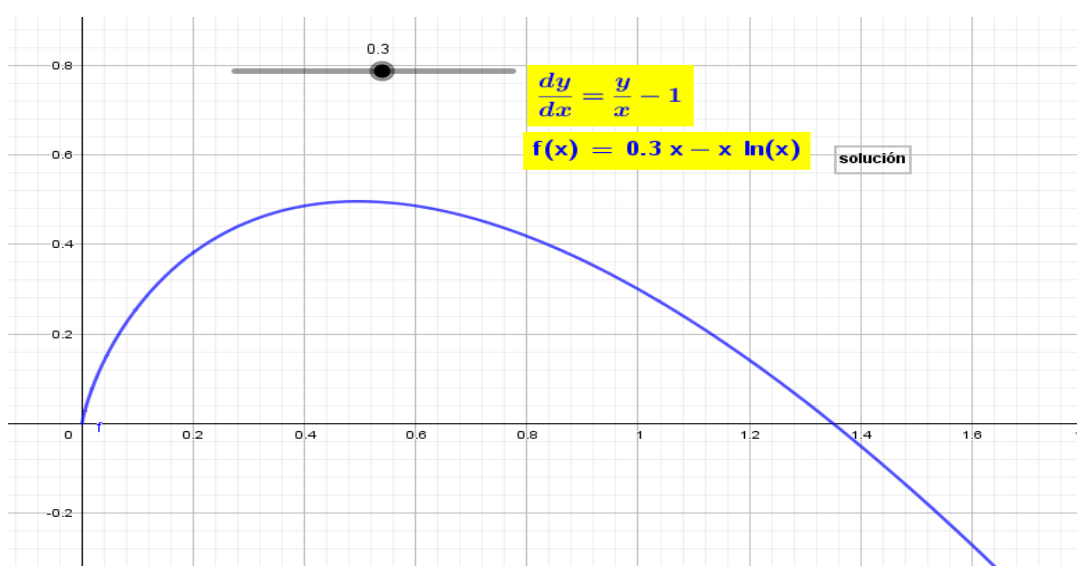

Figure 3

Example 4

$\mathrm{Be}$

$$
\frac{d y}{d x}=\frac{x}{y}-\frac{y}{x}
$$

whose solution is shown in blue in Figure 4 , and $-x^{4}-x^{2}+2 y^{2}=\mathrm{c}$ where "c" move between (-5) and 5.

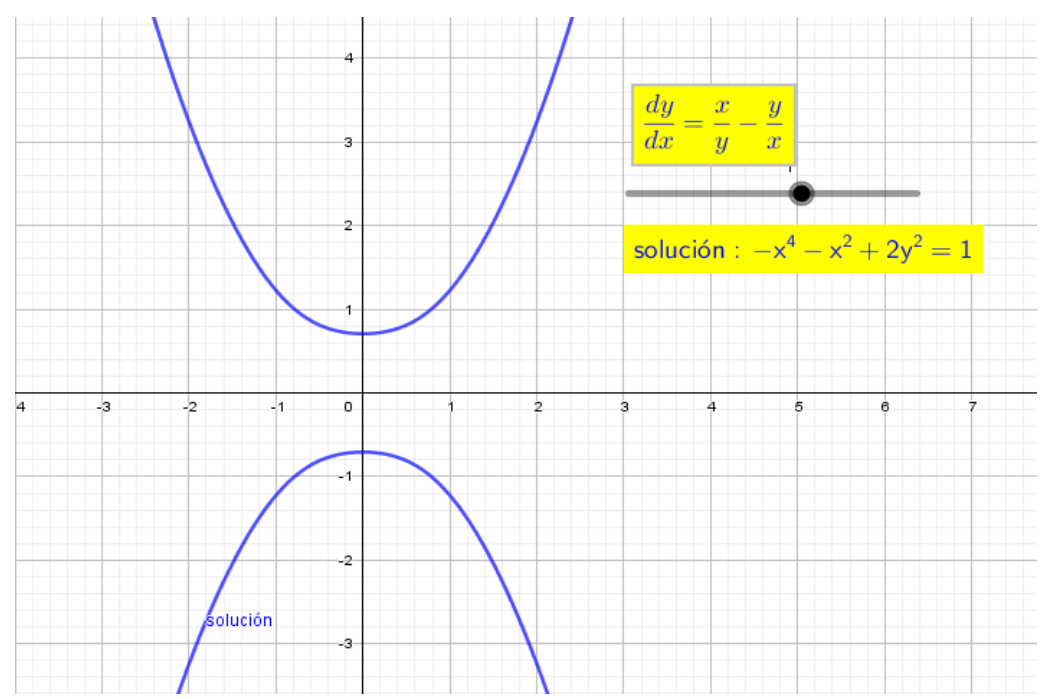

Figure 4 
Example 5

$\mathrm{Be}$

$$
\frac{d y}{d x}=\left(\frac{y}{x}\right)^{2}-a \frac{y}{x}
$$

whose solution is shown in blue in Figure 5 and it is $y_{s}=\frac{(a+1) x}{a c x^{a+1}+c x^{a+1}+1}$

where "a" and "c" move between (-5 )and 5.

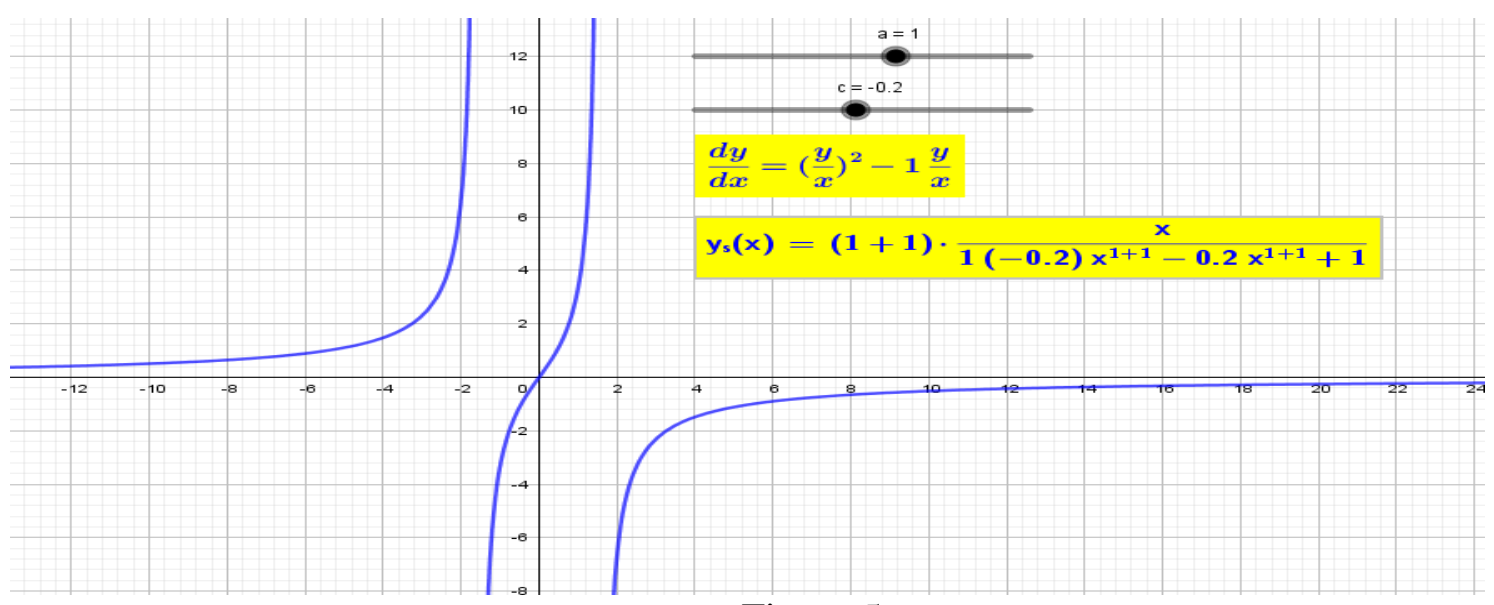

Figure 5

\section{Conclusion}

In this work we wanted to show the various GeoGebra applets as an aid in the teaching process of homogeneous reducible differential equations.

As mentioned in (1), (2) and (3). GeoGebra is of vital importance in the process of learning mathematics and we hope that in a near future that GeoGebra can be used more frequently in the various courses and levels of mathematics at the University Antofagasta.

\section{Acknowledgment}

This paper was supported by the Colloquium of mathematics and the group in teaching and research of the Universidad de Antofagasta-Chile.

\section{References}

Jorge Olivares, Elvis Valero. Exploring the exact differential equations with GeoGebra software, 2021 J. Phys.: Conf. Ser. 1730012063. 
Jorge Olivares, Elvis Valero. Animations and interactive creations in linear differential equations of first order: the case of Geogebra. 2018 J. Phys.: Conf. Ser. 1141012126.

Jorge Olivares, Elvis Valero. Resolving non-homogeneous linear differential equations using the undetermined method coefficients and variation of parameters by means of GeoGebra .2019 J. Phys.: Conf. Ser. 1391012057 